\title{
$\beta$-Glucosidase and cellulase formation by a Trichoderma reesei mutant defective in constitutive $\beta$-glucosidase formation
}

\author{
Josef Strauss $\dagger$ and Christian P. KubiceK* \\ Abteilung für Mikrobielle Biochemie, Institut für Biochemische Technologie und Mikrobiologie, TU Wien, \\ Getreidemarkt 9, A-1060 Austria
}

(Received 8 December 1989; revised 22 February 1990; accepted 14 March 1990)

\begin{abstract}
A mutant of Trichoderma reesei QM 9414 - T. reesei M8 - was isolated after $\gamma$-irradiation. It did not form $\beta$-glucosidase during growth on glycerol or glucose, but secreted $\beta$-glucosidase upon growth on cellobiose. The mutant was also able to grow on cellulose and secrete $\beta$-glucosidase, but with a longer lag. $\beta$-Glucosidase activity could be induced in the mutant in a replacement medium by cellobiose and $\beta$-methyl $D$-glucoside. Mycelia lacking $\beta$-glucosidase were able to take up both these inducers immediately, indicating the presence of a constitutive permease. The mutant produced cellulases upon growth on lactose and - after a lag - on cellulose. Mycelia of $T$. reesei $\mathrm{M8}$ pregrown on glycerol or cellobiose could be induced by sophorose to produce cellobiohydrolase $I$ in a replacement system. The findings show that (i) cellobiose and other $\beta$-linked disaccharides can be taken up by T. reesei without prior hydrolysis, and (ii) that cellobiose most probably induces $\beta$-glucosidase formation during growth on cellulose.
\end{abstract}

\section{Introduction}

The enzymology and genetics of the cellulase system secreted by the hypercellulolytic fungus Trichoderma reesei have been the subject of intense study because of the commercial potential of this system (Enari \& NikuPaavola, 1987; Knowles et al., 1988). Four of the enzymes involved (cellobiohydrolase I and II, EC 3.2.1.91; endoglucanase I and III, EC 3.2.1.4) have already been well characterized; however, the enzyme catalysing the final hydrolysis of cellobiose to glucose, cellobiase ( $\beta$-glucosidase, EC 3.2.1.21), has received less attention. Although its molecular properties have recently been established (Chirico \& Brown, 1987; Schmid \& Wandrey, 1987; Hofer et al., 1989) and the molecular relationship between the extracellular, cellwall-bound (Kubicek, 1981) and plasma-membranebound (Umile \& Kubicek, 1986) forms have been assessed (Hofer et al., 1989), little is known about the regulation of its formation. Sophorose - the most potent inducer of T. reesei cellulases (Mandels et al., 1962) - does not concomitantly induce $\beta$-glucosidase and even represses its formation at high concentrations (Sternberg \& Mandels, 1979, 1980). These authors also reported that

$†$ Present address: Institut de Microbiologie, Université Paris Sud, 91405 Orsay, France.

Abbreviation: CBH I, cellobiohydrolase I. the enzyme is formed constitutively, and that only $\beta$ methyl D-glucoside promoted a fourfold induction (Sternberg \& Mandels, 1982). Jackson \& Talburt (1988) claimed that the $\beta$-glucosidase from $T$. reese $i$ may be involved in cell-wall metabolism during conidiogenesis, and thus not be a true component of the cellulolytic enzyme system. On the other hand, the constitutive $\beta$ glucosidase appears to be involved in the induction of cellulase by cellulose (Kubicek, 1987).

In the present paper we report the isolation of a mutant of Trichoderma reesei QM 9414 that is defective in constitutive formation of $\beta$-glucosidase. We have used this mutant to investigate the regulation of $\beta$-glucosidase biosynthesis in this fungus and its role in cellulase biosynthesis and disaccharide uptake.

\section{Methods}

\footnotetext{
Organism and growth conditions. Trichoderma reesei QM 9414 and mutant $M 8$ derived from it (see below) were used throughout this study. They were maintained on malt-agar slants. Inocula were prepared by harvesting conidia of 14-d-old cultures in sterile tap water containing $0.1 \%$ Tween 80 . The conidia were added to $200 \mathrm{ml}$ medium in widemouthed 1 litre Erlenmeyer flasks to a final concentration of $10^{8}$ conidia $1^{-1}$. The fungi were grown in flasks on a rotary shaker $(250$ r.p.m.) at $28^{\circ} \mathrm{C}$ in the medium described by Mandels \& Andreotti (1978), except that the pH of the medium was kept at 5.0 with $50 \mathrm{mM}$ phosphate/citrate buffer (Labudova \& Farkas, 1983). Carbon sources used are indicated for the respective experiments.
} 
Isolation of mutants defective in constitutive $\beta$-glucosidase formation. A suspension $(5 \mathrm{ml})$ of $10^{7}$ conidia $\mathrm{ml}^{-1}$ was sealed in an appropriate small glass vial and subjected to $\gamma$-irradiation for $25 \mathrm{~s}$ at $25 \mathrm{~kW}$. Thereafter, the suspension was kept at $40^{\circ} \mathrm{C}$ for $72 \mathrm{~h}$ to allow a decay in radioactivity before it was plated on cultivation medium (Mandels \& Andreotti, 1978) containing glycerol as sole carbon source $\left(5 \mathrm{~g} \mathrm{l}^{-1}\right)$, solidified with $2.5 \%(\mathrm{w} / \mathrm{v})$ agar. Since this treatment resulted in a killing rate of approximately $99 \%$ (optimized before), $0.1 \mathrm{ml}$ samples (containing $10^{4}$ putative survivors) were streaked on each plate. $\beta$ Glucosidase activity of individual colonies was assessed by overlaying 2-3-d-old plates (when defined colonies had just developed) with $10 \mathrm{ml}$ $1 \%(\mathrm{w} / \mathrm{v})$ agar in $50 \mathrm{~mm}$-sodium citrate, $\mathrm{pH} 5$, containing $10 \mathrm{~mm}$ methylumbelliferyl $\beta$-D-glucoside. After $2 \mathrm{~h}$ incubation at $28^{\circ} \mathrm{C}$, plates were inspected under UV light. $\beta$-Glucosidase-negative mutants lacked the typical fluorescent halo around the colonies.

Induction of cellulase and $\beta$-glucosidase formation in mycelia of $T$. reesei. Mycelia were pregrown in the medium described above with glycerol as carbon source, and induced to form cellulases or $\beta$ glucosidase in a replacement medium as described by Sternberg \& Mandels (1982) using sophorose or $\beta$-methyl D-glucoside as inducers. Mycelia were cultivated in replacement medium in $100 \mathrm{ml}$ Erlenmeyer flasks containing $10 \mathrm{ml}$ of culture suspension, agitated on a rotary shaker at 200 r.p.m. at $28{ }^{\circ} \mathrm{C}$ for the appropriate time. Mycelial density in the replacement medium at the beginning of the experiment was $1 \mathrm{~g}$ dry wt $1^{-1}$. The initial inducer concentrations in the medium were 1 and $3 \mathrm{~mm}$ for sophorose and $\beta$-methyl D-glucoside, respectively.

During some experiments, uptake of the inducer was followed throughout the incubation. For this purpose, samples $(0.25 \mathrm{ml})$ of the culture broth were collected at intervals, centrifuged in an Eppendorf centrifuge (15 min), and the supernatant analysed for sophorose or $\beta$ methyl D-glucoside by the phenol/sulphuric acid method (Dubois et al., 1956).

Determination of biomass concentration. Biomass concentration was determined by filtering appropriate samples from the culture broth through Gl sintered funnels, washing the mycelial mat with a twofold volume of tap water, followed by a twofold volume of distilled water, and then drying the mat to constant weight at $105^{\circ} \mathrm{C}$. For cellulosecontaining media, intracellular protein was extracted and measured as a biomass equivalent essentially as described previously (Kubicek, 1981).

Assay of enzyme activities. Enzyme activities were assayed either in the culture supernatant [obtained by filtering the culture broth through a Gl sintered funnel, followed by centrifugation $(5 \mathrm{~min})$ in an Eppendorf centrifuge] in the case of endoglucanase, or in unfiltered culture broth to measure the total amount of secreted (i.e. cellmembrane-bound, cell-wall-bound and truly secreted) $\beta$-glucosidase. Endoglucanase and $\beta$-glucosidase were assayed as described by Kubicek (1981), with carboxymethylcellulose (Serva) and $p$-nitrophenyl $\beta$-D-glucoside, respectively, as substrates. One unit $(1 \mathrm{U})$ of enzyme activity is that which released $1 \mu \mathrm{mol}$ glucose equivalent $\min ^{-1}$. Specific activities were expressed as units of activity (mg protein $)^{-1}$. Protein was estimated by the Coomassie Blue binding method (Bradford, 1976).

Electrophoretic techniques. For qualitative demonstration of individual cellulases in the culture supernatant, samples from the culture broth were subjected to SDS-PAGE, followed by Western blotting to nitrocellulose, and immunological detection as described previously (Kubicek et al., 1987). Monoclonal antibodies CH-6 and BG-1 (Mischak et al., 1989; Hofer et al., 1989) were used to detect cellobiohydrolase I and $\beta$-glucosidase, respectively, on the blots.

\section{Results}

\section{Mutant isolation procedure.}

By the outline in Methods, we attempted to isolate $T$. reese $i$ mutants devoid of $\beta$-glucosidase activity when grown on glycerol or glucose as a carbon source. However, yield was very poor: such mutants appeared with a frequency of $0.03 \%$ among the survivors. Various strategies to enrich the desired mutants were tried: however, the fact that the conidia of the fungus already contain $\beta$-glucosidase (Kubicek et al., 1988), which cannot be released by surface active agents (unpublished results), impaired the use of filtration enrichment on cellobiose as a carbon source. Also, the use of $\beta$-Dglucosides of potentially toxic aromatic compounds, e.g. $p$-nitrophenyl $\beta$-D-glucoside, was unsuccessful. Although $p$-nitrophenol severely inhibited growth of $T$. reesei, selection on medium containing this compound led to an exclusive enrichment of $p$-nitrophenol-resistant mutants without enrichment of $\beta$-glucosidase-negative colonies. Therefore, visual inspection after overlaying with methylumbelliferyl $\beta$-D-glucoside was the only successful selection strategy during this work.

From several potential $\beta$-glucosidase-defective isolates, after several subcultivations only a single stable mutant (M8) was obtained. This was used for further investigations.

\section{Effect of carbon source on growth and $\beta$-glucosidase formation by $T$. reesei $Q M 9414$ and $M 8$}

$T$. reesei $\mathrm{QM} 9414$ and the mutant $\mathrm{M} 8$ were compared for growth and $\beta$-glucosidase formation on various carbon sources (Table 1): both strains grew in a comparable manner on all carbon sources tested, the only exception

Table 1. Growth and $\beta$-glucosidase formation by $T$. reesei QM 9414 and M8 on various carbon sources

The concentration of all carbon sources was $0.5 \%(w / v) . \beta$ Glucosidase activity is given as total (i.e. both myceliumassociated and released) extracellular activities per $\mathrm{ml}$ of culture volume. Numbers in parentheses indicate the respective amount of mycelial protein present at the time of harvest (in mg per litre of culture volume). All samples were harvested after $30 \mathrm{~h}$ growth except for M8 on cellulose, which was harvested after $80 \mathrm{~h}$. Values are given for a single experiment only, but repetition yielded consistent results.

\begin{tabular}{llr}
\hline \hline & \multicolumn{2}{c}{$\beta$-Glucosidase activity } \\
\cline { 2 - 3 } $\begin{array}{l}\text { Carbon } \\
\text { source }\end{array}$ & QM 9414 & \multicolumn{1}{c}{ M8 } \\
\hline Glucose & $0.080(152)$ & $<0.010(124)$ \\
Glycerol & $0.080(143)$ & $<0.010(118)$ \\
Lactose & $0.060(86)$ & $0.030(132)$ \\
Cellobiose & $0.300(162)$ & $0.370(154)$ \\
Cellulose & $0.230(65)$ & $0.140(45)$ \\
\hline \hline
\end{tabular}



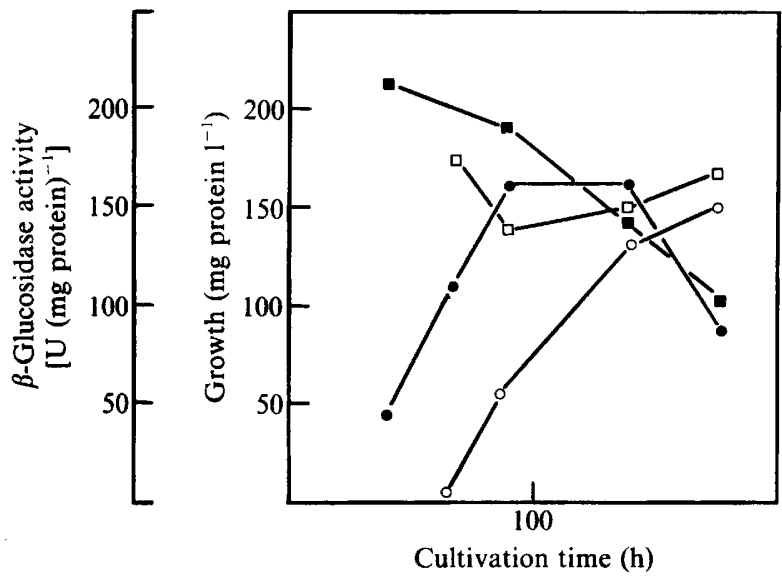

Fig. 1. Growth (O) and $\beta$-glucosidase ( $\square$ ) formation by $T$. reese QM 9414 (filled symbols) and M8 (open symbols) on crystalline cellulose $\left(5 \mathrm{~g}^{-1}\right)$. The medium was prepared according to Mandels \& Andreotti (1978), but peptone was omitted. Growth is given in $\mathrm{mg}$ mycelium-associated protein per litre of culture volume.

being that M8 exhibited a significantly longer lag on cellulose. The specific $\beta$-glucosidase activity of M8 was significantly lower than that of QM 9414 on glucose, glycerol and lactose, but $\beta$-glucosidase was clearly present upon cultivation on cellobiose. Growth of M8 on cellulose (in the absence of peptone) exhibited a considerable lag but was subsequently comparable, and $\beta$-glucosidase was also formed (Fig. 1). These results indicate that we have apparently isolated a mutant of $T$. reesei with a defect in a regulatory region rather than a $\beta$-glucosidase structural gene.

Interestingly, we found that the $\beta$-glucosidase formed by QM 9414 and M8 was exclusively cell-wall and cellmembrane-bound during cultivation on all carbon sources except lactose and cellulose. During growth on these two compounds, $10-30 \%$ of the total external $\beta$ glucosidase activity appeared in the culture supernatant (unpublished data).

\section{Induction of $\beta$-glucosidase formation in $T$. reesei QM 9414 and $M 8$}

$\beta$-Methyl D-glucoside has been reported to be an inducer of $T$. reesei $\beta$-glucosidase (Sternberg \& Mandels, 1982). Since M8 still produced $\beta$-glucosidase during growth on cellobiose, we precultivated the two strains of $T$. reesei on glycerol, and investigated the induction of $\beta$-glucosidase by transferring them to medium containing either $\beta$ methyl D-glucoside or cellobiose. Both inducers promoted $\beta$-glucosidase formation, albeit at a lower rate with cellobiose (31.5 and 11.7 versus 54.2 and $18.3 \mathrm{U} \mathrm{ml}^{-1}$ for $\beta$-methyl D-glucoside and cellobiose as inducers with strains QM 9414 and mutant M8, respectively). Although the mycelium of $T$. reesei M8 contained virtually

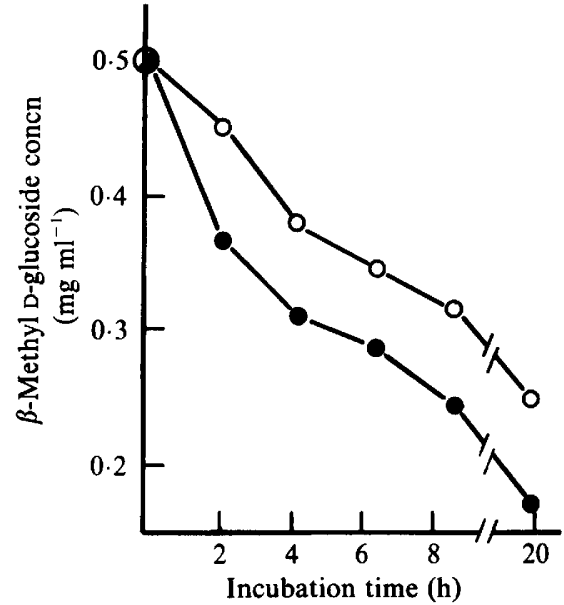

Fig. 2. Uptake of $\beta$-methyl D-glucoside by $T$. reesei $Q M 9414(O)$ and M8 (O) during induction of $\beta$-glucosidase in a replacement system, using glycerol-pregrown mycelium.

no $\beta$-glucosidase activity, it took up $\beta$-methyl D-glucoside without a lag (Fig. 2). The same kinetics were also observed with cellobiose (C. Fritscher \& C. P. Kubicek, unpublished data). These results therefore demonstrate that $\mathrm{M} 8$, although defective in constitutive $\beta$-glucosidase formation, can be induced to form this enzyme. The fact that the two inducers used are apparently taken up by the $\beta$-glucosidase-devoid mycelium indicates the presence of a constitutive permease for these sugars in $T$. reesei.

\section{Regulation of formation of cellobiohydrolase I by T. reesei M8}

T. reesei $\mathrm{QM} 9414$ secretes a cellulase enzyme system into the culture supernatant when it is grown on cellulose or on lactose as inducing (cellulose) and carbon-catabolitederepressing (lactose) carbon sources, respectively (Kubicek et al., 1990). Since $\beta$-glucosidase has been implicated in the formation of the true inducer during cellulose degradation (Gritzali \& Brown, 1979; Vaheri et al., 1979; Kubicek, 1987), we investigated whether mutant M8 can produce cellulases on both these carbon sources. Cellobiohydrolase I (CBH I), which accounts for up to $60 \%$ of the secreted cellulase protein, was measured as a 'marker' of cellulase formation during these experiments. Fig. 3 shows Western blots of culture filtrates from M8, indicating that lactose led to apparently normal CBH I formation; the pattern of CBH I secretion during growth of M8 on cellulose paralleled that of growth and $\beta$-glucosidase formation, exhibiting a similar lag.

In order to find out whether mutant M8 can be induced to form CBH I by sophorose in a normal manner, resting cell experiments with glycerol- and 


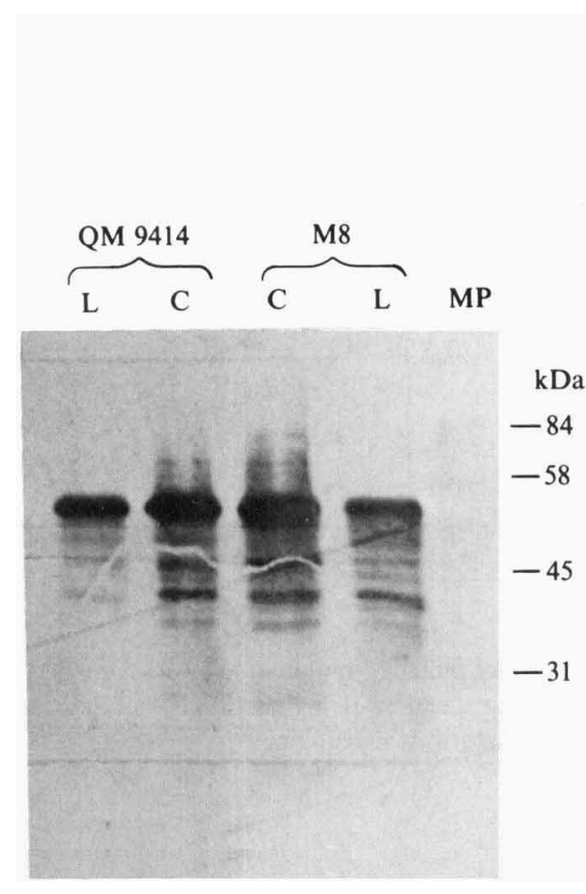

Fig. 3

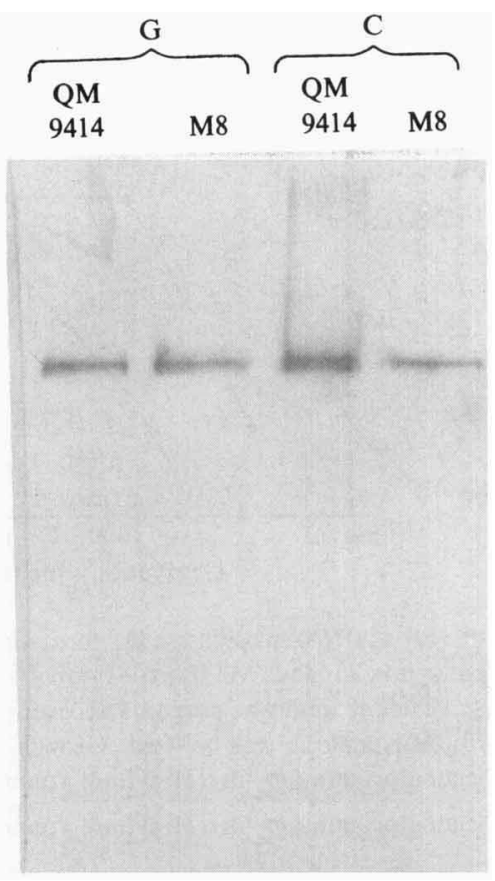

Fig. 4

Fig. 3. Immunodetection of CBH I in culture broth of $T$. reesei QM 9414 and M8, after growth on lactose (L) and cellulose (C) for $60 \mathrm{~h}$ (M8 on cellulose for $120 \mathrm{~h}$ ). Samples $(10 \mu \mathrm{l}$ of culture broth, $2-4 \mu \mathrm{g}$ protein) were subjected to SDS-PAGE and Western blotting as described in Methods, and stained by using monoclonal antibody CH-6 (Mischak et al., 1989). The positions of prestained marker proteins (MP) are indicated.

Fig. 4. Immunodetection of CBH I in the culture broth of sophorose-induced mycelia of $T$. reesei QM 9414 and M8, pregrown on cellobiose (C) and glycerol (G). Samples ( $10 \mu l$ of culture broth, $0 \cdot 5-1 \mu \mathrm{g}$ protein) were taken from cultures that had been exposed to the inducer for $20 \mathrm{~h}$ and processed as described in the legend to Fig. 3.

cellobiose-pregrown mycelia were done. Fig. 4 indicates that $\mathrm{CBH}$ I was induced in mycelia by growth on both carbon sources. Also the same time-course of induction was observed with both mycelia (data not shown). These findings are in accordance with those reported earlier (Kubicek, 1987) and show that $\beta$-glucosidase is not necessary for cellulase formation when sophorose is used as an inducer.

\section{Discussion}

The prime goal of the present investigation was the isolation of a $\beta$-glucosidase-negative mutant of $T$. reesei. However, we observed that such mutants apparently occur at a very low frequency. We do not know whether this is due to a very low level of expression of the $\beta$ glucosidase gene or to an as yet unknown essential function of $\beta$-glucosidase for $T$. reesei, such as an involvement in morphogenesis (Jackson \& Talburt, 1988) which would render a mutation in the $\beta$ glucosidase gene lethal. Cloning of $\beta$-glucosidase from this fungus is now in progress in at least two laboratories (T. Berges, C. Barreau \& J. Begueret, conference poster,
Vienna, 1989; R. L. Mach \& C. P. Kubicek, unpublished), which should provide an answer to this important question.

One mutant, defective in constitutive formation of $\beta$ glucosidase, was isolated. While the properties of the mutant are consistent with the existence of two different $\beta$-glucosidase genes in $T$. reesei, one constitutive and one inducible, recent results comparing the identity of the plasma-membrane-bound (constitutive) $\beta$-glucosidase with the cell-wall-bound and the extracellular $\beta$-glucosidase from this fungus contradict this idea (Hofer et al., 1989). At present, no proof is available for the existence of a second secreted $\beta$-glucosidase from $T$. reesei. Hence we conclude that we have isolated a mutant altered or defective in the regulation of $\beta$-glucosidase formation.

Studies on the induction of cellulase formation in the mutant M8 showed that it grew only poorly on cellulose and also secreted little cellulase into the medium. In contrast, cellulase levels comparable to strain QM 9414 were secreted in a replacement medium upon induction by sophorose. These findings stress the importance of $\beta$ glucosidase for inducing cellulases in $T$. reesei during growth on cellulose - most probably by forming the inducer - as shown recently (Kubicek, 1987); further- 
more, they show that $T$. reesei apparently possesses a transport system for $\beta$-linked disaccharides. Our studies on the uptake of cellobiose and $\beta$-methyl D-glucoside by mycelia of M8 pregrown on glucose or glycerol (such mycelia lack $\beta$-glucosidase) indicate that this transport system is constitutive and has apparently not been altered in M8; hence the slow growth of the mutant on celluose may be due to uptake of cellobiose instead of extracellular hydrolysis. Recent studies from our laboratory show that the in vivo activity of the permease is less than one-tenth of that of total extracellular $\beta$-glucosidase (C. Fritscher, R. Messner \& C. P. Kubicek, unpublished results).

The permease may also be involved in regulating $\beta$ glucosidase activity: in the present paper, we have provided evidence that both cellobiose and $\beta$-methyl Dglucoside act as physiological inducers of $\beta$-glucosidase. In view of the existence of a constitutive uptake system, this may provide a mechanism by which the cell avoids overaccumulation of cellobiose under conditions which the intracellular $\beta$-glucosidase (Inglin et al., 1980) cannot handle. Hence elevated intracellular cellobiose levels may induce increased extracellular cellobiose hydrolysis.

$\beta$-Glucosidase has often been considered not to be a component of the cellulase system of $T$. reesei because it is differentially regulated. In contrast, the present results suggest that cellulases and $\beta$-glucosidase are both components of a regulatory network appropriate for economic cellulose hydrolysis : the constitutive $\beta$-glucosidase may function in the induction of cellulases by formation of sophorose from cellobiose (supplied by the constitutive cellulases; Kubicek et al., 1988), whereas additional $\beta$-glucosidase may become induced upon increased cellobiose formation by the induced cellulases. Such a model also suggests that the amount of $\beta$ glucosidase secreted by $T$. reesei may depend on the activity of the intracellular $\beta$-glucosidase described by Inglin et al. (1980). Represssion of formation of extracellular $\beta$-glucosidase (Sternberg \& Mandels, 1980) and induction of the intracellular $\beta$-glucosidase (Loewenberg, 1984) by sophorose is in accordance with this view. This may offer strategies for finding $\beta$ glucosidase-overproducing mutants of $T$. reesei in the future.

This work was supported by Fond zur Förderung Wissenschaftlicher Forschung, P 7231 BIO. The authors are thankful to Dr F. Grass and Dipl.Ing. B. Schwabl for their help with $\gamma$-irradiation.

\section{References}

BRADFORD, M. M. (1976), A rapid and sensitive method for quantitation of microgram quantities of protein utilizing the principle of protein dye-binding. Analytical Biochemistry 72, 248254.
Chirico, W. J. \& Brown, R. D., JR (1987). Purification and characterization of $\beta$-glucosidase from Trichoderma reesei. European Journal of Biochemistry 165, 333-341.

Dubois, M., Gilles, K. A., Hamilton, J. K., Rebers, P. A. \& Smith, F. (1956). Colorimetric method for determinations of sugars and related substances. Analytical Chemistry 28, 350-356.

EnARI, T. M. \& NikU-PaAvola, M. L. (1987). Enzymatic hydrolysis of cellulose: is the current theory of the mechanisms of hydrolysis valid. CRC Critical Reviews in Biotechnology 5, 67-87.

GritZali, M. \& Brown, R. D., JR (1979). The cellulase system of Trichoderma: relationship between purified extracellular enzymes from induced and cellulose grown cells. Advances in Chemistry Series 181, 237-260.

Hofer, F., Weissinger, E., Mischak, H., Messner, R., MeiXnerMonori, B., BlaAs, D., Visser, J. \& KubiceK, C. P. (1989). A monoclonal antibody against the alkaline extracellular $\beta$-glucosidase from Trichoderma reese $i$ : reactivity with other Trichoderma $\beta$ glucosidases. Biochimica et Biophysica Acta 992, 298-306.

Inglin, M., Feinberg, B. A. \& Loewenberg, J. R. (1980). Partial purification and characterization of a new intracellular $\beta$-glucosidase of Trichoderma reesei. Biochemical Journal 185, 515-519.

JaCkson, M. A. \& TAlbURT, D. E. (1988). Mechanisms for $\beta$ glucosidase release into cellulose-grown Trichoderma reesei culture supernatants. Experimental Mycology 12, 203-216.

Knowles, J., Teeri, T. T., Lehtovaara, P., Pentillä, M. \& SAloheImo, M. (1988). The use of gene technology to investigate fungal cellulolytic enzymes. In Biochemistry and Genetics of Cellulose Degradation, pp. 153-169. London \& New York: Academic Press.

KUBICEK, C. P., (1981). Release of carboxymethyl-cellulase and $\beta$ glucosidase from cell walls of Trichoderma reesei. European Journal of Applied Microbiology and Biotechnology 13, 226-231.

KUBICEK, C. P. (1987). Involvement of a conidial endoglucanase and a plasma-membrane-bound $\beta$-glucosidase in the induction of endoglucanase synthesis by cellulose in Trichoderma reesei. Journal of General Microbiology 133, 1481-1487.

Kubicek, C. P., Panda, T., Schrefert-Kunar, G., Gruber, F. \& MESSNER, R. (1987). O-linked and N-linked glycosylation is necessary for the secretion of endoglucanases I and II by Trichoderma reesei. Canadian Journal of Microbiology 33, 698-703.

Kubicek, C. P., MüHLberger, G., Grotz, M., John, E. \& KubiceKPranz, E. M. (1988). Properties of a conidial-bound cellulase system of Trichoderma reesei. Journal of General Microbiology 134, 12151222.

Kubicek, C. P.,Messner, R., Fritscher, C., Strauss, J. \& KubicekPranz, E. M. (1990). Regulatory aspects of formation and secretion of cellulases by Trichoderma reesei. In Trichoderma Cellulases: Biochemistry, Physiology, Genetics and Production. Edited by C. P. Kubicek, D. E. Eveleigh, H. Esterbauer, W. Steiner \& E. M. Kubicek-Pranz. London: Technical Communications (in the Press).

Labudova, I. \& Farkas, V. (1983). Multiple forms in the cellulase system of Trichoderma reesei during its growth on cellulose. Biochimica et Biophysica Acta 744, 135-140.

LOEWENBERG, J. R. (1984). Sophorose induction of an intracellular $\beta$ glucosidase in Trichoderma. Archives of Microbiology 137, 53-57.

Mandels, M. \& ANDReotri, R. E. (1978). Problems and challenges in the cellulose to cellulase fermentation. Process Biochemistry 13, 6-13.

Mandels, M., Parrish, F. W. \& Reese, E. T. (1962). Sophorosē as an inducer of cellulase in Trichoderma viride. Journal of Bacteriology 83 , 400-408.

Mischak, H., Hofer, F., Messner, R., Weissinger, E., Hayn, M., Tomme, P., Esterbauer, H., Küchler, E., Claeyssens, M. \& KUBICEK, C. P. (1989). Monoclonal antibodies against different domains of cellobiohydrolase I and II from Trichoderma reesei. Biochimica et Biophysica Acta 990, 1-7.

SCHMID, G. \& WANDREY, C. (1987). Purification and partial characterization of a cellodextrin glucohydrolase ( $\beta$-glucosidase) from Trichoderma reesei strain QM 9414. Biotechnology and Bioengineering 30, 571-585.

SternBerG, D. \& MANDELS, G. R. (1979). Induction of cellulolytic enzymes in Trichoderma reesei by sophorose. Journal of Bacteriology 139, 761-769. 
SternBerg, D. \& MANDELS, G. R. (1980). Regulation of the cellulolytic system of Trichoderma reese $i$ by sophorose : induction of cellulase and repression of $\beta$-glucosidase. Journal of Bacteriology 144, 1197-1199.

STERNBERG, D. \& MANDELS, G. R. (1982). $\beta$-Glucosidase induction and repression in the cellulolytic fungus Trichoderma reesei. Experimental Mycology 6, 115-124.
UMile, C. \& KubiceK, C. P. (1986). A constitutive, plasma-membrane bound $\beta$-glucosidase in Trichoderma reesei. FEMS Microbiology Letters 34, 291-295.

Vaheri, M. P., Leisola, M. \& Kaupinnen, V. (1979). Transglycosylation products of the cellulase system of Trichoderma reesei. Biotechnology Letters 1, 41-46. 